\title{
Joseph Cornell et les Surréalistes à New York : Dalí, Duchamp, Ernst, Man Ray...
}

Lyon, Musée des Beaux-Arts, du 8 octobre 2013 au 10 février 2014

\section{Anne Reynès-Delobel}

\section{(2) OpenEdition}

\section{Journals}

Édition électronique

URL : https://journals.openedition.org/transatlantica/6449

DOI : $10.4000 /$ transatlantica. 6449

ISSN : 1765-2766

Éditeur

Association française d'Etudes Américaines (AFEA)

Référence électronique

Anne Reynès-Delobel, « Joseph Cornell et les Surréalistes à New York : Dalí, Duchamp, Ernst, Man

Ray... », Transatlantica [En ligne], 1 | 2013, mis en ligne le 19 février 2014, consulté le 04 février 2023

URL : http://journals.openedition.org/transatlantica/6449 ; DOI : https://doi.org/10.4000/

transatlantica.6449

Ce document a été généré automatiquement le 4 février 2023

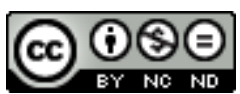

Creative Commons - Attribution - Pas d'Utilisation Commerciale - Pas de Modification 4.0 International - CC BY-NC-ND 4.0

https://creativecommons.org/licenses/by-nc-nd/4.0/ 


\section{Joseph Cornell et les Surréalistes à New York : Dalí, Duchamp, Ernst, Man Ray...}

Lyon, Musée des Beaux-Arts, du 8 octobre 2013 au 10 février 2014

\section{Anne Reynès-Delobel}

Remerciements à Sylvaine Manuel de Conssinguy, chargée des relations presse, Mairie de Lyon et à Géraldine Chouard pour sa relecture et ses suggestions.

"What kind of man is this, who from old brown cardboard photographs collected in second-hand

bookstores has reconstructed the nineteenth century 'grand tour' of Europe for his mind's eye more vividly than those who took it, who was not born then and has never been abroad [...], who in full consciousness of twentieth-century plasticity of Arp, of Giacometti, of André Breton, of the cubist collage, of surrealism, and the precision of

a Yankee instrument-maker - precision of feeling, that is, can incorporate this sense of the past in something that could only have been conceived of at present, that will remain one of the presents of the present to the future - what kind of man indeed?"

C'est sur cet hommage rendu en 1953 par Robert Motherwell à Joseph Cornell (1903-72) que se clôt l'exposition présentée au Musée des Beaux-Arts de Lyon jusqu'au 10 février 2014. Cette manifestation, la première consacrée à l'artiste américain dans un musée français depuis plus de trente ans, se propose de faire découvrir son travail en le situant dans le contexte des échanges artistiques transatlantiques entre 1930 et 1950, années de maturité de l'œuvre de Cornell, et plus précisément ceux générés par le surréalisme qui connaît alors une phase de diffusion importante à New York, grâce à 
l'entremise déterminante de galeristes, marchands d'art, collectionneurs et conservateurs, tels Julien Levy, Peggy Guggenheim, Alfred J. Barr et A. Everett Austin. Le but n'est toutefois pas d'assimiler Cornell au groupe surréaliste, mais à l'inverse de mettre en lumière son apport au surréalisme et, de manière plus générale, de rappeler la place de premier plan qu'occupe son œuvre dans la production artistique du $\mathrm{XX}^{\mathrm{e}}$ siècle, en particulier après la Seconde Guerre Mondiale.

2 L'exposition s'ouvre sur une présentation du surréalisme, compris non pas comme un mouvement et encore moins comme une école, mais comme un "réseau » au sein duquel la personnalité de Joseph Cornell s'est développée. En adoptant cette approche, les deux commissaires, Sylvie Ramond et Matthew Affron, poursuivent un double objectif. En effet, s'ils ont à cœur de souligner la manière dont la démarche surréaliste, de par l'importance qu'elle accorde au rêve et au jeu dans la production d'analogies poétiques, souvent inconscientes, a permis à Cornell d'affirmer et de diversifier ses propres pratiques, essentiellement tournées vers le collage et les processus qui y sont associés (montage, construction, assemblage), ils tiennent encore davantage à faire sentir que l'artiste américain ne s'identifie pas purement et simplement au surréalisme, mais demeure constamment à sa marge. C'est d'ailleurs en revendiquant cette position marginale que Cornell entendait développer, par le biais de ce qu'il appelait sa « magie blanche" (en contraste avec l'onirisme noir privilégié par les surréalistes), des possibilités «encore plus saines» que celles poursuivies par les autres membres du groupe. En ce sens, le propos premier de l'exposition est de présenter l'œuvre de Joseph Cornell comme l'un des surgeons du surréalisme, tout en explorant ses filiations historiques plus complexes, en mettant en valeur ses spécificités intrinsèques (notamment la mise en place d'une méthode personnelle très élaborée de l'archivage ou encore le lien privilégié avec la photographie et le cinéma) et en insistant sur l'idée que l'œuvre se construit et demeure dans une sorte «d'entre-deux » entre la France et les États-Unis, entre le jouet et le cabinet de curiosités, et entre le musée d'histoire naturelle et le musée d'art. Comme le suggère l'emprunt à la déclaration de Motherwell, l'idée est moins d'enfermer l'artiste dans un «-isme » et son œuvre dans une boîte étiquetée (no pun intended) que de lui conserver sa part fondamentale de secret et d'énigme.

3 L'entreprise, qui relève à bien des égards du tour de force, a été menée à bien grâce au temps consacré au travail préparatoire par les commissaires et leurs collaborateurs (six années), au nombre et à la diversité des œuvres présentées (plus de deux cent cinquante, issues de collections françaises et étrangères, publiques et privées) et à une politique d'accrochage qui fait découvrir certains aspects de l'activité de Cornell très rarement montrés, notamment sa création cinématographique, en les mettant en résonance avec le reste de son œuvre. Associant logique thématique et progression chronologique, le parcours se déploie en dix moments qui mettent l'accent sur un aspect particulier de la pratique de l'artiste (boîtes, collages, objets, films) en écho avec les activités du réseau surréaliste pris au sens large. On y retrouve ainsi, outre les principales figures de la scène européenne en exil à New York, d'illustres précurseurs comme Duchamp ou des artistes périphériques du surréalisme regroupés sous le terme de « Néo-Romantiques».

4 Dès la première salle, le visiteur peut mesurer visuellement la richesse et l'intensité des échanges au sein du réseau formé à Paris par les artistes, poètes et écrivains surréalistes, au cours des deux décennies qui précèdent leur exil à New York, à l'orée 
des années quarante. Une galerie photographique composée d'une vingtaine de portraits réalisés par Man Ray et Lee Miller, parmi lesquels plusieurs tirages argentiques d'époque, réunit les principaux acteurs et compagnons de route du surréalisme (Breton, Duchamp, de Chirico, Ernst, Tanguy, Dalí, Gala et Buñuel) dans des mises en scènes tantôt drolatiques (comme dans ces célèbres portraits de Marcel Duchamp en Rrose Sélavy et d'André Breton en nonne, signés de Man Ray et datés respectivement de 1921 et 1924), tantôt poétiques: deux autoportraits de Joseph Cornell réalisés en collaboration avec Lee Miller et extraits de la série Twelve Needles Dancing on the Point of an Angel (1933) juxtaposent ainsi le profil de l'artiste à un modèle réduit de goélette auquel sont accrochés une longue mèche de cheveux blonds, une boule et un papillon. Outre qu'elle fait écho à l'un des premier collages de Cornell, Untitled (Schooner) (1931), cette photographie évoque sa passion pour le montage et l'assemblage, et révèle une affinité manifeste avec les expérimentations sur l'objet menées à la même époque par les surréalistes, thématique explorée en détail dans une autre partie de l'exposition.

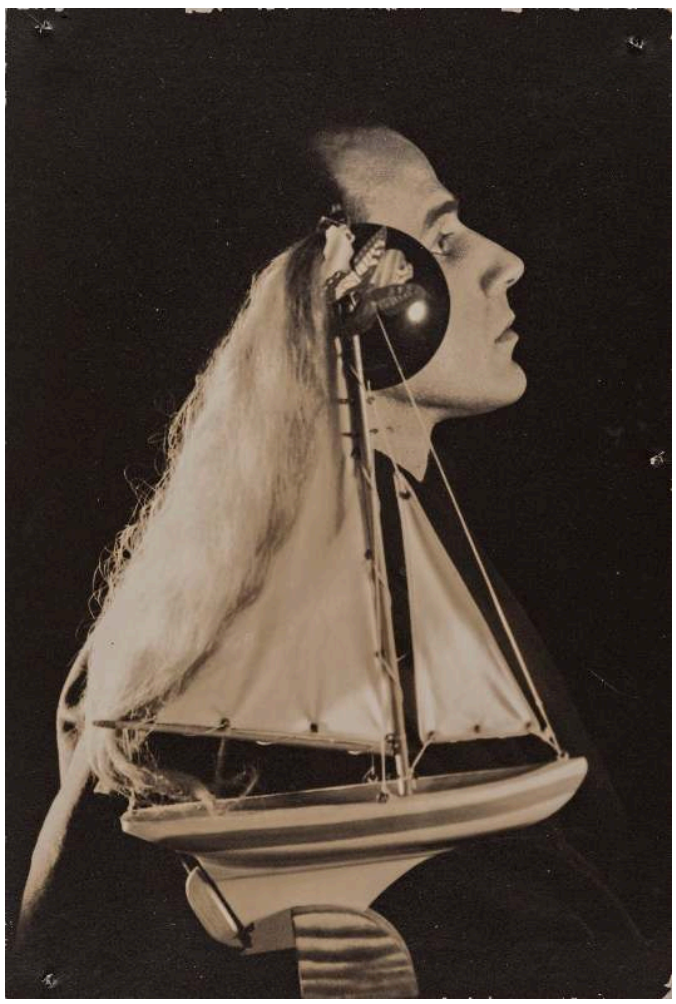

Lee Miller, Portrait of Joseph Cornell, 1932- 1933 @ Adagp, Paris 2013

5 L'image de la figure de proue évoquée par le montage semble suggérer, quant à elle, que moins de deux ans après sa première rencontre avec Julien Levy (également photographié par Miller avec son épouse Joella dans leur galerie, point de rayonnement du surréalisme outre-Atlantique), Cornell était reconnu comme un membre de premier plan du groupe surréaliste. Si Julien Levy a été un acteur déterminant dans la carrière de l'artiste, en lui donnant notamment l'occasion d'exposer ses collages lors de l'exposition Surréalisme de janvier 1932, un autre portrait de Miller rappelle le rôle non moins crucial joué par Arthur Everett (Chick) Austin, autre «passeur » du surréalisme aux États-Unis, à l'origine de l'exposition Newer Super Realism de 1931 qui présenta pour 
la première fois les œuvres de Dalí, de Chirico, Ernst, Masson, Picasso et Pierre Roy au public américain.

6 Egalement signés de Lee Miller, quatre tirages argentiques d'époque intitulés Objects by Joseph Cornell (glass dome) permettent de faire remonter l'utilisation de l'objet trouvé et l'introduction du verre (sous la forme de cloches de verre) dans l'œuvre de Cornell à 1932, soit plusieurs années avant l'apparition des boîtes vitrées qui formeront un aspect essentiel de sa production à partir de 1936 et jusqu'au début des année soixante. Ces boîtes, ainsi que les « minutiae " (petite boîtes à pilules enfermant de minuscules objets trouvés) et les coffrets plats et vitrés parfois appelés " pocket objects », sont présentés dans la deuxième salle, dont l'impeccable scénographie introduit le visiteur au cœur de l'imaginaire singulier de l'artiste. Dans une demi-pénombre invitant à l'émerveillement et favorisant l'attention aux menus détails, les boîtes de Cornell livrent leurs trésors (boutons, cailloux, coquillages, ressorts, poudres et sables colorés, etc.) et dévoilent une quête passionnée de la poésie du quotidien. En invitant le point de vue de la conservatrice Colette Vicat-Blanc, l'audioguide aiguise l'attention sur la composition de ces "petits mondes très travaillés", tout de délicatesse et d'équilibre. Une vidéo mettant en scène un prestidigitateur ganté de blanc permet par ailleurs de montrer que certains objets ont été conçus par Cornell en vue d'être manipulés, tel ce Flat SandBox de 1950, qu'il suffit d'incliner de quelques degrés pour libérer toute une charge poétique et émotionnelle. Ce dispositif, allusion à la "magie blanche" de l'artiste, souligne l'importance primordiale du toucher (et plus encore du désir de toucher) dans toute sa production. Il est repris avec bonheur en plusieurs autres endroits de l'exposition. Plus loin, un autre petit objet, Bel Echo Gruyère (qui produit un meuglement quand on le retourne, selon un procédé depuis exploité à satiété par le commerce) souligne avec humour l'importance du son, autre élément important du travail de Cornell. Quant à ce livre-objet de 1940-42, il introduit un procédé, également récurrent chez l'artiste, consistant à détourner l'objet livre en l'évidant pour créer du volume et démultiplier les lectures possibles. Essentielles pour la compréhension du reste du parcours, les deux boîtes intitulées Ephemera Metaphysics: Novalis (1941) et Hölderlin Object (1944-46) mettent en lumière la sensibilité particulière de Cornell pour le romantisme allemand qui est, avec la poésie française du XIXe siècle, une source d'inspiration inépuisable. Enfin, dialoguant avec les objets placés dans les vitrines, les collages et photographies accrochés aux murs révèlent l'importance du geste du recyclage chez l'artiste américain. À ce propos, la série de Lee Miller déjà évoquée, Objects by Joseph Cornell (glass dome), montre l'état initial d'une construction recyclée en 1936 dans sa première boîte d'ombre intitulée Untitled (Soap Bubble Set). On y trouve les objets (pipes en terre, verres, gravures, cartes célestes et miroirs) qui vont devenir des éléments fondamentaux du vocabulaire de l'artiste au point de donner lieu à plus de cent autres boîtes construites sur le même thème jusqu'en 1960 .

7 L'exposition propose ensuite une salle entièrement consacrée au collage que Cornell pratique tout au long des années trente et jusqu'au début des années quarante, puis abandonne jusqu'aux années cinquante, période à laquelle il lui est plus difficile de trouver des matériaux européens pour ses boîtes, suite aux restrictions et aux pénuries de l'après-guerre. Une série de collages originaux de Max Ernst conservés au Centre Pompidou rappelle que la rencontre avec le roman-collage La Femme à cent têtes (Editions du Carrefour, 1929) fut déterminante pour le jeune Cornell qui se présenta à Julien Levy, en novembre 1931, avec six montages témoignant d'une étonnante affinité 
avec le travail du surréaliste. S'il demeure une ambiguïté quant à l'influence d'Ernst (s'agit-il d'une véritable filiation ou d'une coïncidence?), les collages de Cornell relèvent d'un même goût pour les images vernaculaires et l'iconographie du XIX ${ }^{\mathrm{e}}$ siècle. Parmi les œuvres issues de collections étrangères, on notera deux collages inédits réalisés par Max Ernst pour Une semaine de bonté (1934), ainsi que ceux d'André Breton, Valentine Penrose, Max Bucaille et Paul Eluard, qui attestent l'idée du réseau surréaliste comme lieu vivace d'échanges et d'émulation. On peut mesurer l'évolution de la production de Cornell en comparant ce collage de 1931-32 (repris par Julien Levy pour la couverture de son livre Surrealism) représentant un garçonnet qui fait s'envoler les lettres du mot «surréalisme » en soufflant dans un cornet de papier, avec les images de la série Lettres d'un alphabet (1933), dédiée à Raymond Radiguet et à l'occasion de laquelle l'artiste renouvelle sa pratique en introduisant la couleur, puis avec America Fantastica, la couverture du numéro spécial de janvier 1943 de View, la revue de Charles Henry Ford, dont l'iconographie ouvertement " américaine » fait se côtoyer le Chrysler Building, King Kong, les Chutes du Niagara, les Indiens, et introduit le personnage imaginaire de Bérénice, petite Américaine de Nouvelle-Angleterre qui occupe une place centrale dans l'imaginaire de Cornell.

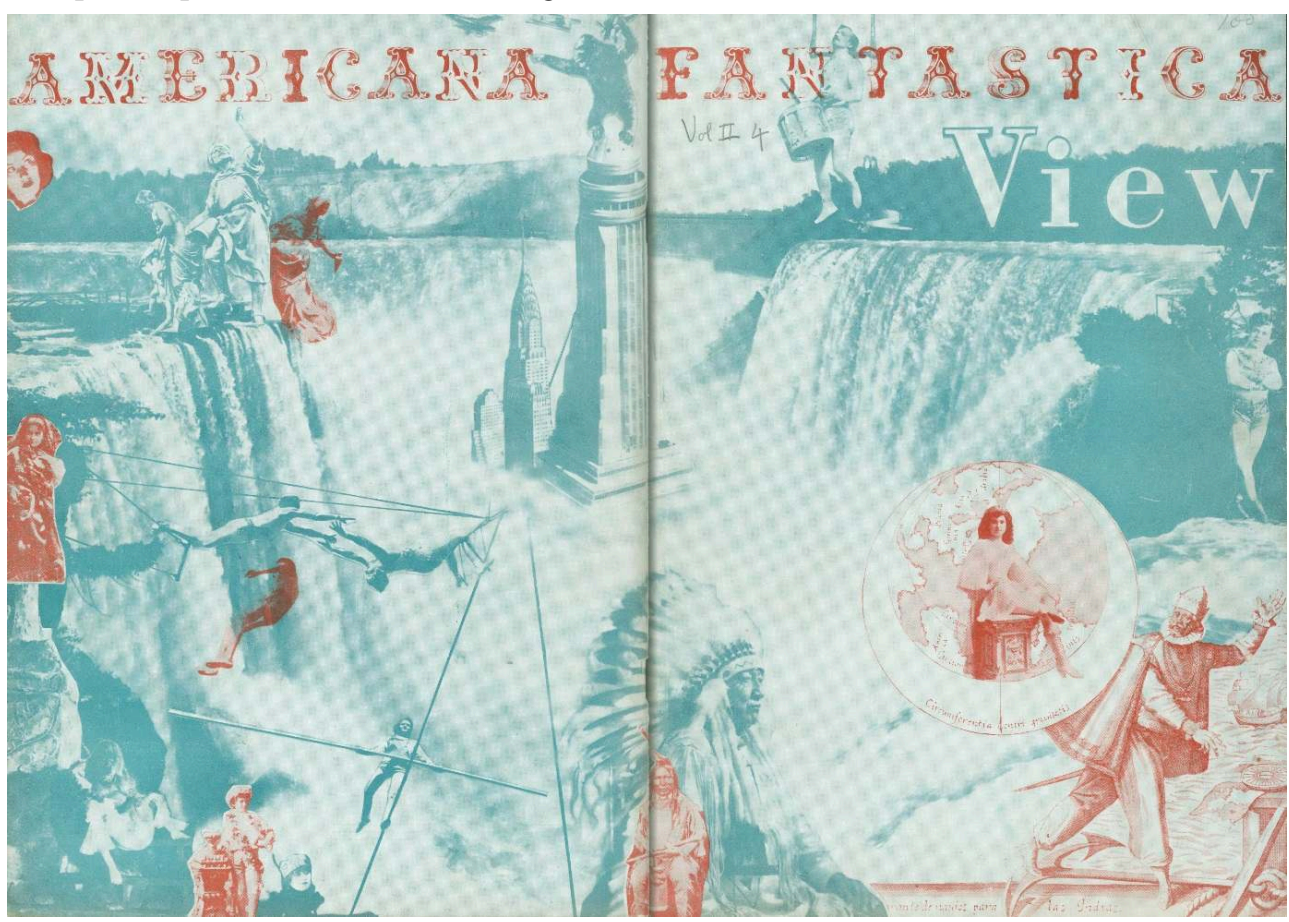

Joseph Cornell, Collage reproduit en couverture du numéro spécial de View, 2e série n 4, janvier 1943, Americana Fantastica. (c) Adagp, Paris 2013

8 L'exploration de la "constellation surréaliste » se poursuit ensuite en deux temps. L'objectif est de montrer plus précisément les aspects du surréalisme retenus par Cornell (certains agencements formels, le goût de la surprise née du décalage des motifs et une prédilection pour le jeu), tout en soulignant sa volonté de rester à la marge du surréalisme pour explorer de nouvelles voies formelles et poétiques. Dans un premier espace, des tableaux de Magritte, de Chirico, Dalí, Tanguy et Mina Loy, ainsi que des frottages de Max Ernst, dialoguent avec une série d'assemblages par Cornell, Ernst et Calder. Si les images "paranoïaques-astrales" de Dalí participent d'une inspiration très différente de celle qui a donné naissance aux figures angéliques de Mina Loy ou aux toiles de Pierre Roy (qui privilégient davantage les effets optiques que 
la démarche surréaliste), on peut toutefois relever dans ces œuvres un intérêt commun pour l'enfance, le secret, la mélancolie, ainsi que pour les objets en équilibre ou en mouvement, éléments que l'on retrouve dans Untitled (Tilly Tosh), une boîte de Cornell réalisée vers 1935 et qui a inspiré l'affiche de l'exposition lyonnaise. À l'étage, la démonstration continue de manière convaincante autour de la notion d'objet, "fait surréaliste par excellence », dont on rappelle qu'il était au centre de la pensée de Breton dès 1924 et orientera les expérimentations du groupe à partir de 1931. La pensée de Freud, autre inspiration marquante du surréalisme, est également évoquée. Ici encore, les objets exposés font écho aux photographies de May Ray et Lee Miller qui ont largement contribué à les faire connaître. Ainsi, les assemblages de la série "Rêveobjet » d'André Breton photographiés par Man Ray entrent en résonnance directe avec les objets «à fonctionnement symbolique » regroupés au centre de la pièce. Témoins d'une fascinante communauté de pensée, ces œuvres de Man Ray, Dalí, Breton et Cornell participent, à travers la reprise d'un certain nombre d'éléments (comme le métronome, l'œil ou la cloche de verre), d'une volonté d'extraire l'objet quotidien de sa logique utilitaire pour "objectiver l'activité du rêve, son passage dans la réalité " (Breton). L'accent mis sur le mouvement rappelle la proximité essentielle avec le jeu et propose ainsi une transition fluide avec les trois salles suivantes, consacrées au cinéma et aux images en mouvement.

9 La partie consacrée aux films-collages de Cornell constitue un autre point fort de l'exposition. D'une part, parce que cet aspect de l'œuvre, rarement montré, est ici largement mis en valeur, d'autre part, parce qu'il est parfaitement intégré à l'accrochage. Cinéphile averti, grand amateur et collectionneur des films des frères Lumière, Méliès, Ferdinand Zecca ou Emile Cohl, Cornell devient en décembre 1936 un acteur du monde du $7^{\text {ème }}$ art lorsque Julien Levy présente son film-collage, Rose Hobart, dont les images, extraites d'un mélodrame d'aventure du réalisateur George Melford, East of Borneo (1931), ont été tirées de leur séquence d'origine et projetées à travers un filtre bleu, tandis que la bande-son originale était remplacée par de la musique latine et la vitesse de projection ralentie. Le film qui en résulte, long de dix-neuf minutes, invite à une expérience sensorielle des plus étonnantes. D'autres films courts, muets ou sonores, des années trente et quarante (achevés dans les années soixante par Larry Jordan d'après les consignes de Cornell) permettent d'associer la passion de l'artiste pour le cinéma avec son goût pour les divertissements populaires comme les tours de prestidigitation, la fête foraine ou le vaudeville. Tous procèdent en outre de répétitions et d'effets de boucle que l'on trouve également à l'œuvre dans les films expérimentaux de Marcel Duchamp (Anemic Cinema, 1926) et de Man Ray et Robert Desnos (L'Etoile de mer, 1928), ce qui démontre chez l'artiste américain un refus d'établir une discrimination entre culture populaire et expérimentations d'avant-garde. Cet intérêt de Cornell pour la cinétique et l'illusion transparaît dans son utilisation récurrente du jouet et établit un lien intéressant avec un autre pan de son œuvre.

10 La référence à Duchamp et à son exploration des gadgets optiques (rotoreliefs et disques optiques) se prolonge chez Cornell avec l'utilisation de jouets en vente dans le commerce, comme le zootrope, le thaumatrope et autres artefacts optiques, tel le phénakisticope et le stéréoscope, dont l'artiste faisait également la collection. L'exposition évoque cette passion pour explorer plusieurs autres dimensions de son travail. Elle démontre ainsi la façon dont le procédé de l'image en mouvement a présidé à la fabrication de plusieurs assemblages, comme par exemple cette boîte-jouet au titre 
en français, "Le Voyageur dans les glaces ", Jouet Surréaliste (1935), composée de sept disques comportant chacun deux images distinctes et d'un mécanisme pour les mettre en mouvement, sur le principe du thaumatrope. Dans une autre construction, Beehive (Thimble Forest), réalisée entre 1943 et 1948, qui met en scène de petits dés à coudre montés sur des épingles, le mécanisme de l'illusion d'optique s'appuie sur un jeu de miroirs pour produire cette fois-ci un effet proche du zootrope. Il suffit en effet de regarder cet assemblage par un petit trou d'observation pour que se produisent un changement d'échelle et une mise en abyme rendant inutile le recours au nombre et à la mesure: le minuscule devient démesurément grand, tandis que l'image se démultiplie à l'infini. Il est aussi intéressant de remarquer que le jeu sur la perception visuelle se double souvent d'une dimension tactile et sonore. Ainsi, le petit assemblage de Beehive (Thimble Forest) se met à vibrer au moindre mouvement imprimé à la boîte. Ailleurs, comme avec Object Fenetre (1937), de petites boîtes contenant de minuscules objets produisent un bruit, à chaque fois différent, lorsqu'on les secoue, sauf une, d'inspiration très "cagienne » avant la lettre, qui ne renferme que du silence. De construction en construction, on comprend de quelle manière la poétique de Cornell se fonde sur la dimension matérielle des processus optiques, invitant ainsi la manipulation. Ceci explique en partie pourquoi ses boîtes intègrent si souvent des daguerréotypes, dont les images varient, suivant l'angle d'inclinaison, du négatif au positif, voire mélangent les deux. De manière plus générale, l'utilisation d'artefacts optiques comme le zootrope, le thaumatrope ou le stéréoscope souligne le lien que ces jouets, très en vogue à l'époque victorienne, entretiennent avec le XIX ${ }^{\mathrm{e}}$ siècle, pour lequel l'artiste avait une sensibilité particulière. Baudelaire et sa "Morale du joujou » sont à cette occasion convoqués pour rappeler le rôle crucial du jouet dans la sensibilisation artistique, mais aussi, et peut-être surtout pour Cornell, « la tendance métaphysique » de l'enfant. Enfin, elle établit un lien entre les effets liés aux jouets optiques (absence de linéarité, agitation visuelle, répétitions et reprises en boucles, soudaineté de l'action) et les processus utilisés dans les films-collages de l'artiste. Grâce à cette lecture opérée par les commissaires, le visiteur est invité à apprécier l'œuvre dans toute sa diversité, tout en restant attentif à sa profonde cohérence et à son originalité intrinsèque.

11 La même logique préside à l'exploration de l'influence déterminante de l'œuvre de Duchamp que l'exposition choisit de présenter sous la forme du binôme «Cornell/ Duchamp ». La longue complicité qui liait les deux artistes (elle débute en 1933 et ne s'achèvera que trente-cinq ans plus tard avec la disparition de Duchamp) autorise la mise en relation étroite de certaines de leurs œuvres. Cornell, qui assista Duchamp pour la fabrication de onze boîtes de la série $\mathrm{A}$ et de vingt-cinq boîtes de la série $\mathrm{B}$ de la Boîte-en-valise (achevée en 1941), a sans doute tiré parti de cette collaboration pour assembler certains projets d'envergure, comme The Crystal Cage (Portrait of Berenice), fruit d'un long (1943-1960) et patient travail d'accumulation et d'archivage non-linéaire de toutes sortes de documents visant à récréer la figure imaginaire d'une jeune Américaine. Il est intéressant de rappeler que l'idée d'une esthétique partagée par Cornell et Duchamp avait été exploitée dès le début des années quarante par deux expositions organisées dans les galeries de Peggy Guggenheim et Julien Levy, à New York. Toutefois, on aurait aimé que l'exposition prenne aussi le soin de souligner la spécificité individuelle de la démarche de chaque artiste. Par exemple, si la mise en relation critique de l'idée de « reproduction » avec celle d' " original » et la dimension autoréflexive de l'acte artistique sont essentielles dans la réflexion de Duchamp, elles le 
sont beaucoup moins dans celle de Cornell qui privilégie la citation, l'emprunt, la reprise ou le détournement, notamment des œuvres de la littérature française dont l'artiste est féru (celles de Nerval, Mallarmé, Roussel, Saint Exupéry et Alain-Fournier sont quelques-unes de ses références-clés). Par ailleurs, le parallèle établi entre certaines œuvres (Pharmacie, 1914, de Duchamp / Pharmacy, 1943, de Cornell) semble un peu forcé et peinera peut-être à convaincre. II ressort néanmoins de cette mise en rapport avec Duchamp plusieurs idées primordiales pour la compréhension de l'œuvre de Cornell : il apparaît clairement qu'elle relève d'un travail d'assemblage très contrôlé et d'un état de rêverie permanente qui la distinguent de l'automatisme surréaliste. On perçoit également qu'elle recèle une part de secret qui résiste à toute tentative d'explication et d'interprétation.

12 Ces notions sont développées dans la salle suivante qui traite des affinités de Cornell avec le travail d'artistes occupant comme lui une position en marge du surréalisme et qu'il découvre en 1933, lors de l'exposition Twenty-Five Years of Russian Ballet, à la galerie Julien Levy. Ces artistes, Eugene et Leonid Berman, Pavlev Tchelitchev, Christian Bérard et Kristians Tonny, ne forment pas un mouvement à proprement parler, mais sont néanmoins connus depuis 1926, année où leurs œuvres sont présentées à la galerie Druet, à Paris, sous le nom de "Néo-Romantiques", inventé par le critique Waldemar George. L'exposition chez Julien Levy et celle qui se tient plus tard à la galerie Hugo encouragent Cornell à orienter sa pratique (sous forme de dossiers, collages et boîtes) autour de sa passion pour la danse. Dès le début des années vingt, il a pu voir Isadora Duncan et Martha Graham se produire dans l'East Village. Toutefois, c'est le ballet romantique et la figure mythique des grandes ballerines du XIX siècle qui retiennent toute son attention. De la même façon qu'il consacre de nombreuses boîtes aux stars du $7^{\text {ème }}$ art (Greta Garbo, Lauren Bacall, Rose Hobart), il dédie des « bouquets d'hommage » à Tamara Toumanova, Maria Taglioni, Fanny Elssler, Carlotta Grisi et Fanny Cerrito. En 1944 , ces quatre dernières forment d'ailleurs le sujet de la couverture qu'il réalise pour la revue Dance Index, Le quatuor dansé à Londres. La participation de l'artiste à la revue lancée en 1942 par Lincoln Kirstein, ainsi qu'à d'autres largement diffusées (Vogue, Good Housekeeping, Harper's Bazaar), de même que sa fréquentation assidue des Archives de la danse au MoMA (également inaugurées par Kirstein en 1939), font de Cornell l'une des personnalités les plus érudites du monde de la danse à New York. L'exposition présente notamment un dossier intitulé Portrait of Ondine (1940), témoin de l'attachement de l'artiste pour cette mythique figure hybride, intensément mélancolique, qui cristallise sa passion pour le répertoire russe (Balanchine, Lifar) et la littérature française (Nerval, Gauthier, Giraudoux). La mélancolie, le rêve et la métamorphose reviennent dans les toiles et les costumes des artistes présentés ici, dans un fascinant jeu d'échos. La scénographie vise à révéler les affinités stylistiques et thématiques qui relient ces œuvres, peu connues et rarement montrées, tout en conservant leur part de mystère. À cet égard, l'univers fantastique et onirique de Leonor Fini, dont l'exposition présente quatre toiles, ne manquera pas d'arrêter le visiteur.

13 La dixième et dernière étape du parcours, évoque l'évolution de la production de Cornell après la Seconde Guerre Mondiale et le retour des surréalistes en France. Ce départ, ainsi que la difficulté qu'il éprouve à se procurer des matériaux venus d'Europe, expliquent en partie son retour au collage et son recours fréquent au recyclage d'anciennes boîtes, comme les emblématiques Soap Bubble Sets (dont l'exposition présente ici deux versions recyclées, Soap Bubble Set/Figurehead/Pipe datée de 1955-60 et Untitled (Soap Bubble Set, Latitude and Longitude Box), datée de 1960) ou les Medici Slot 
Machines (Medici Boy, 1953).Toutefois, un renouvellement en profondeur de sa pratique se lit également dans la création de séries aux lignes plus épurées et dont le contenu, moins anecdotique et moins "littéraire", donne lieu à une inspiration plus personnelle. Commencée en 1950, la série Hotels recompose une géographie imaginaire qui invite le spectateur à une navigation singulière. Les éléments qui forment le vocabulaire familier de l'artiste (extraits d'ouvrages en français et de revues de cinéma, cartes postales) sont assemblés dans des environnements baignés d'une froide lueur bleue évoquant des constellations lointaines (Andromède, Cassiopée) ou les profondeurs marines. La série des Dovecotes, elle aussi initiée en 1950, procède d'un arrangement abstrait des lignes, formes et volumes qui semble annoncer les bouleversements du pop art ou du minimalisme. Ces assemblages aux rythmes géométriques et symétriques reposent sur le principe de la grille. Leur austérité formelle est contrebalancée par leur texture qui stimule le désir du toucher: leur surface est en effet souvent enduite d'une épaisse couche de peinture blanche, semblable à de la chaux, que l'artiste a vieillie artificiellement pour y faire apparaître des pelures et des craquelures. Cette tactilité est encore renforcée par la présence de boules blanches ou de divers objets hétéroclites (Dovecote, 1953) que la main a envie de saisir et de palper, voire de déranger et de réarranger. À travers la série des Hotels et celle des Dovecotes, le traitement du temps et de la solitude prennent un tour plus aigu que partout ailleurs dans l'œuvre. Comme pour rendre sensibles les préoccupations formelles qui infléchissent le travail de Cornell à cette période, les commissaires ont choisi de placer ces œuvres (pour la plupart des boîtes) au centre de la salle, à hauteur de regard, ce qui permet au visiteur d'en faire le tour et d'en apprécier toute la matérialité. Les inscriptions au dos de certaines constructions livrent des indices sur leurs titres successifs, ainsi que sur les divers lieux où elles ont pu être exposées. On notera à ce propos qu'après la guerre, Cornell se rapproche de la galerie Egan qui montrera son travail à plusieurs reprises, notamment la série Aviary, en 1949. L'exposition s'achève donc à l'orée des années cinquante sur l'idée que Cornell, tout en revendiquant plus que jamais ses sources d'inspiration et ses pratiques propres, est un artiste totalement en prise avec l'art de son temps, notamment avec l'expressionisme abstrait qui se développe depuis quelques années sous l'impulsion de Jackson Pollock.

Le parcours se referme sur la photographie, agrandie à la dimension d'un mur, d'une partie des archives de Cornell et sur la déclaration de Motherwell citée en préambule. On n'aurait pu trouver meilleure manière d'évoquer l'impression laissée par cet itinéraire au cœur de l'œuvre de Cornell, dans la mesure où cette dernière relève à la fois d'un travail d'assemblage extrêmement savant et minutieusement contrôlé, et d'une méditation personnelle appartenant à « un ordre privé qui ne concède au public que des échos respectant le secret initial», comme le note Gérard Durozoi dans son Histoire du mouvement surréaliste (2004). On sera également sensible à la remarque de Motherwell qui place la dimension «Yankee » du travail du reclus d'Utopia Parkway du côté d'un "précisionnisme sentimental " dont bien des facettes restent encore à explorer. C'est donc sur le rivage de la curiosité que nous dépose cette exposition d'un bout à l'autre passionnante.

15 En marge de la manifestation, un catalogue abondamment illustré propose une dizaine d'essais mettant en lumière les principaux aspects de l'œuvre de Cornell. Leur lecture permet notamment de mesurer la foisonnante érudition qui a étayé la production de l'artiste et donné lieu à une pratique de l'archivage que Judie Hauptmann n'hésite pas à comparer à la méthode du Konvolut mise en œuvre par Walter Benjamin. On retiendra 
également l'étude de Stephen Bann, qui défend la prééminence de la notion de " curiosité » chez Cornell en établissant un parallèle avec la grande tradition des cabinets de collectionneurs, et celle d'Emmanuel Guigon, qui rappelle le contexte de création de «l'objet surréaliste » et insiste lui aussi sur la part de secret irréductible dans les objets produits par Cornell. Les commissaires, Sylvie Ramond et Matthew Ramond, livrent également deux articles très intéressants, respectivement consacrés à la place du musée (et notamment du "dime museum ») dans l'activité artistique de Cornell et à sa production cinématographique (notamment en lien avec la photographie, autre pan essentiel de sa collection). Une réflexion poétique de Patrick Mauriès sur les " Néo-Romantiques " permet d'en apprendre davantage sur cet autre réseau en marge du surréalisme, tandis qu'un glossaire établi par Anne Théry propose une utile série de coups de projecteur sur les relations de Cornell à la France.

Un colloque international sera organisé les 7 et 8 février 2014 à Paris (Institut National de l'Histoire de l'Art) et à Lyon (Musée des Beaux-Arts). Le programme sera mis en ligne sur le site du musée http://www.mba-lyon.fr/mba/sections/fr/expositions-musee/ cornell-surrealistes/expo-cornell-lyon/. Au printemps 2014, une version plus réduite de l'exposition voyagera aux États-Unis pour être présentée au Fralin Museum of Art de l'Université de Virginie.

\section{AUTEUR}

\section{ANNE REYNÈS-DELOBEL}

Université Aix-Marseille 